\title{
GPS as a base for analysis of perturbations of space based and ground based applications
}

\author{
Tsvetelina Traykova ${ }^{1, *}$, and Veselin Radkov ${ }^{2}$ \\ ${ }^{1}$ CAMO Partner Ltd., Sofia, Bulgaria \\ ${ }^{2}$ Technical University - Sofia, Bulgaria
}

\begin{abstract}
The present work is dedicated to examining the impact of respective Navstar/GPS embarrass impact on the running of the global navigation system as well as synthesizing a computer program for their impact judgment as well. Two major groups of perturbation factors are addressed: perturbations proven impact on the orbital segment and perturbations proven impact on the running of the user's piston ring (nonorbital disturbances); it is being synthesized in the theoretical productions on the ground, plan code that yes on is modeled. The influence of those who embarrass the invoices on the accuracy of specifying the location.
\end{abstract}

\section{Introduction}

NAVSTAR, commonly known as Global Position System (GPS), provides worldwide three-dimensional position and velocity information to users on the Earth's surface or in the air, including precise time. In order to determine true user position, the data have to be transmitted continuously with high accuracy and perturbation effects to be minimized.

The purpose of this paper is to introduce an algorithm for computation of the errors in determining the user position, which are caused by different perturbation effects. For instance GPS satellite orbits are influenced by perturbation of factors such as gravity, oblateness of Earth, atmospheric drag, solar radiation pressure, etc. Other factors that cause an error are Satellite clock error, Relativistic Effects, Ionosphere effect, Tropospheric delay, Multipath effect affect, etc.

The presented algorithm is implemented in a computer code that allows for the analysis of the influence of the different factors mentioned above - one by one, or in a combination, on determining the user position. Numerical simulations were performed as well and the results of the analysis will be presented in a successive paper.

\section{Perturbations at GPS satellite orbits}

To include the effects of the perturbations, the equation of motion is written in a general form as [1]:

$$
\frac{d^{2} r}{d t^{2}}=-\mu \frac{r}{r^{3}}+a_{p},
$$

where $r$ is position vector; $\mu=G M_{e},\left(M_{e}-\right.$ Earth mass, $G=6,6726( \pm 0,0005) \cdot 10^{-11} \mathrm{~m}^{3} \mathrm{~kg}^{-1} \mathrm{~s}^{-2}-$ gravitational constant; $a_{p}$ - the resultant vector of all the perturbing accelerations. The magnitude of the $a_{p}$ in the Solar system for all types of satellite orbits is 10 times smaller than the central force, or $\left|a_{p}<<\mu r / r^{3}\right|$. The resultant vector may consist of several types of perturbing accelerations, described in Table 1.

Perturbations at satellite orbit can be classified as conservative and non-conservative. For conservative accelerations, $a_{p}$ is an explicit function of position only, and there is no net energy transfer, i.e. the mean semimajor axis of the orbit is constant. The second type, nonconservative, such as atmospheric drag and tidal friction effect, $a_{p}$ is an explicit function of both position and velocity, i.e. energy transfer occurs. As a result, the mean semi-major axis of the orbit changes.

Perturbations at satellite orbit which are included in the present research are described in this work.

Table 1. Perturbation accelerations.

\begin{tabular}{|l|l|}
\hline \multicolumn{1}{|c|}{ Gravitational: } & \multicolumn{1}{|c|}{ Non-gravitational: } \\
\hline Third-body (sun/moon) attractions & The non-spherical Earth \\
\hline $\begin{array}{l}\text { Outgassing (fuel tank leaks on the } \\
\text { spacecraft) }\end{array}$ & Atmospheric drag \\
\hline & Solar-radiation pressure \\
\hline & Tidal friction effect \\
\hline
\end{tabular}

\subsection{Gravity perturbation}

Each Earth satellite experiences the force of gravitational attraction from the other bodies in the system - three-body system; Sun, Moon, Earth. Forces exerted on body 1 by bodies 2 and 3 are $F_{12}$ and $F_{13}$, respectively. Likewise, body 2 experiences the forces $F_{21}$ and $F_{23}$ whereas the forces $F_{31}$ and $F_{32}$ act on body 3 [2]:

$$
\begin{aligned}
& F_{12}=-F_{21}=\frac{G m_{1} m_{2}\left(R_{2}-R_{1}\right)}{\left\|R_{2}-R_{1}\right\|^{3}} ; \\
& F_{13}=-F_{31}=\frac{G m_{1} m_{3}\left(R_{3}-R_{1}\right)}{\left\|R_{3}-R_{1}\right\|^{3}} ;
\end{aligned}
$$

\footnotetext{
* Corresponding author: ctraiikova@abv.bg
} 


$$
F_{23}=-F_{32}=\frac{G m_{3} m_{2}\left(R_{3}-R_{2}\right)}{\left\|R_{3}-R_{2}\right\|^{3}} .
$$

With respect to the inertial system the acceleration of the bodies is:

$$
\mathrm{a}_{i}=\ddot{R}_{\iota}, i=1,2,3 \ldots,
$$

where $R_{i}$ is absolute position vector of body $i$.

The equation of motion of body 1 is:

$$
F_{12}+F_{13}=m_{1} a_{1} .
$$

Substituting in (6) equations for $F_{12}$ and $F_{13}$ yields:

$$
a_{1}=\frac{G m_{2}\left(R_{2}-R_{1}\right)}{\left\|R_{2}-R_{1}\right\|^{3}}+\frac{G m_{3}\left(R_{3}-R_{1}\right)}{\left\|R_{3}-R_{1}\right\|^{3}} .
$$

In a similar way the equations for body 2 and 3 are:

$$
\begin{aligned}
& a_{2}=\frac{G m_{1}\left(R_{1}-R_{2}\right)}{\left\|R_{1}-R_{2}\right\|^{3}}+\frac{G m_{3}\left(R_{3}-R_{2}\right)}{\left\|R_{1}-R_{2}\right\|^{3}} ; \\
& a_{3}=\frac{G m_{1}\left(R_{1}-R_{3}\right)}{\left\|R_{1}-R_{3}\right\|^{3}}+\frac{G m_{2}\left(R_{2}-R_{3}\right)}{\left\|R_{2}-R_{3}\right\|^{3}} .
\end{aligned}
$$

The accelerations are related to the velocities by:

$$
\frac{d v_{i}}{d t}=a_{i}, \quad i=1,2,3,
$$

and the position vectors are likewise related to the velocities:

$$
\frac{d R_{i}}{d t}=v_{i}, \quad i=1,2,3 .
$$

Equations (6-9) form a system of ordinary differential equations in the variable time $t$. Since there are no external forces, the acceleration on the center of the masses is zero, i.e.

$$
\begin{aligned}
a_{G} & =0, \\
& \text { so that } \quad \frac{d v_{G}}{d t}=0, \\
& \text { and } \\
\frac{d R_{G}}{d t} & =v_{G} .
\end{aligned}
$$

Since the system above decouples with respect to the first three variables, it is easily integrable - the initial positions $R_{i 0}$ and velocities $v_{i 0}$ are given, to find $v_{i}$ the first three equations have to be integrated. The resulting functions are substituted in the second three equations of the system to obtain $R_{i}$ as a function of time.

The three-body system could be extended to $N$-body system. A geostationary satellite is related to three other bodies, but because of the lower mass of the satellite it is not taken into account in the system.

To solve the above problem the integrations are usually done numerically. For instance, writing all vectors component-wise with respect to $X Y Z$ basis as column vector we obtain the following presentation:

$$
\left\{R_{1}\right\}=\left\{\begin{array}{l}
R_{1 x} \\
R_{1 y} \\
R_{1 z}
\end{array}\right\} ;\left\{R_{2}\right\}=\left\{\begin{array}{l}
R_{2 x} \\
R_{2 y} \\
R_{2 z}
\end{array}\right\} ;
$$

$$
\left\{R_{3}\right\}=\left\{\begin{array}{l}
R_{3 x} \\
R_{3 y} \\
R_{3 z}
\end{array}\right\} ;\left\{R_{G}\right\}=\left\{\begin{array}{l}
R_{G x} \\
R_{G y} \\
R_{G z}
\end{array}\right\},
$$

and

$$
\begin{aligned}
& \left\{v_{1}\right\}=\left\{\begin{array}{l}
v_{1 x} \\
v_{1 y} \\
v_{1 z}
\end{array}\right\} ;\left\{v_{2}\right\}=\left\{\begin{array}{l}
v_{2 x} \\
v_{2 y} \\
v_{2 z}
\end{array}\right\} ; \\
& \left\{v_{3}\right\}=\left\{\begin{array}{l}
v_{3 x} \\
v_{3 y} \\
v_{3 z}
\end{array}\right\} ;\left\{v_{G}\right\}=\left\{\begin{array}{l}
v_{G x} \\
v_{G y} \\
v_{G z}
\end{array}\right\},
\end{aligned}
$$

therefore

$$
\begin{aligned}
& \left\{\mathrm{a}_{1}\right\}=\left\{\begin{array}{l}
\mathrm{a}_{1 x} \\
\mathrm{a}_{1 y} \\
\mathrm{a}_{1 z}
\end{array}\right\}=\left\{\begin{array}{l}
\frac{G m_{2}\left(R_{2 x}-R_{1 x}\right)}{R_{12}}+\frac{G m_{3}\left(R_{3 x}-R_{1 x}\right)}{R_{13}} \\
\frac{G m_{2}\left(R_{2 y}-R_{1 y}\right)}{R_{12}}+\frac{G m_{3}\left(R_{3 y}-R_{1 y}\right)}{R_{13}} \\
\frac{G m_{2}\left(R_{2 z}-R_{1 z}\right)}{R_{12}}+\frac{G m_{3}\left(R_{3 z}-R_{1 z}\right)}{R_{13}}
\end{array}\right\} ; \\
& \left\{\mathrm{a}_{2}\right\}=\left\{\begin{array}{l}
\mathrm{a}_{2 x} \\
\mathrm{a}_{2 y} \\
\mathrm{a}_{2 z}
\end{array}\right\}=\left\{\begin{array}{l}
\frac{G m_{2}\left(R_{1 x}-R_{2 x}\right)}{R_{12}}+\frac{G m_{3}\left(R_{3 x}-R_{2 x}\right)}{R_{13}} \\
\frac{G m_{2}\left(R_{1 y}-R_{2 y}\right)}{R_{12}}+\frac{G m_{3}\left(R_{3 y}-R_{2 y}\right)}{R_{13}} \\
\frac{G m_{2}\left(R_{1 z}-R_{2 z}\right)}{R_{12}}+\frac{G m_{3}\left(R_{3 z}-R_{2 z}\right)}{R_{13}}
\end{array}\right\} ; \\
& \left\{\mathrm{a}_{3}\right\}=\left\{\begin{array}{l}
\mathrm{a}_{3 x} \\
\mathrm{a}_{3 y} \\
\mathrm{a}_{3 z}
\end{array}\right\}=\left\{\begin{array}{l}
\frac{G m_{2}\left(R_{1 x}-R_{3 x}\right)}{R_{12}}+\frac{G m_{3}\left(R_{2 x}-R_{3 x}\right)}{R_{13}} \\
\frac{G m_{2}\left(R_{1 y}-R_{3 y}\right)}{R_{12}}+\frac{G m_{3}\left(R_{2 y}-R_{3 y}\right)}{R_{13}} \\
\frac{G m_{2}\left(R_{1 z}-R_{3 z}\right)}{R_{12}}+\frac{G m_{3}\left(R_{2 z}-R_{3 z}\right)}{R_{13}}
\end{array}\right\} ;
\end{aligned}
$$

where

$$
\begin{aligned}
& R_{12}=\left\|R_{2}-R_{1}\right\| ; \\
& R_{13}=\left\|R_{3}-R_{1}\right\| ; \\
& R_{23}=\left\|R_{3}-R_{2}\right\| .
\end{aligned}
$$

Next, 24-component column vector is formed

$$
\{f\}=\left|\left\{R_{1}\right\}\left\{R_{2}\right\}\left\{R_{3}\right\}\left\{R_{G}\right\}\left\{v_{1}\right\}\left\{v_{2}\right\}\left\{v_{3}\right\}\left\{v_{G}\right\}\right|^{T} .
$$

The derivative of this vector in the corresponding components is a column vector

$$
\left\{\frac{d f}{d t}\right\}=\left|\left\{v_{1}\right\}\left\{v_{2}\right\}\left\{v_{3}\right\}\left\{v_{G}\right\}\left\{a_{1}\right\}\left\{a_{2}\right\}\left\{a_{3}\right\}\{0\}\right|^{T} .
$$

\subsection{Perturbation due to Oblateness of the Earth}

In order to illustrate the idea of gravitational harmonic, the concept of gravitational potentials is introduced. The gravitational field of a certain body mass may be presented by a potential function.

Earth harmonics are the result of gravitational potential using gravitational potential function. Those harmonics are the terms of a mathematical expansion through which the deviations from a sphere can be represented.

The most commonly used model of the Earth is WGS 84. The normally used harmonics are those of the lowest 
order. For example commonly used zonal harmonics are $J_{2}, J_{3}$, and $J_{4}$.

The second harmonic $J_{2}$ is related to the Earth's equatorial oblateness, in reference to Earth rotation and the estimated difference between the polar radius and equatorial radius - about $22 \mathrm{~km}$.

If the mass of the body is uniformly distributed, the potential function is $\Phi$ is

$$
\Phi=\frac{\mu}{r},
$$

where $r$ is geometric range, $\mu=G M_{e}, G$ - gravitational constant, $M_{e}-$ Earth mass.

The potential function including the effect of oblateness of Earth can be presented as

$$
\Phi=\frac{\mu}{r}+R
$$

where $R$ is perturbing function (disturbing function), including $J_{2}$ effect, which can be given either in terms of the orbital parameters as [3]:

$$
R=\frac{\mu J_{2} A_{e}^{2}}{2 r^{3}}\left[1-\frac{3}{2} \sin ^{2} i(1-\cos 2 u)\right] .
$$

Perturbing accelerations due to $J_{2}$ could be given in spherical coordinates as [1]:

$$
\begin{gathered}
F_{r}=-\frac{3}{2} \frac{\mu J_{2} a_{e}^{2}}{r^{4}}\left[1-\frac{3}{2} \sin ^{2} i(1-\cos 2 u)\right] \\
F_{S}=-\frac{3}{2} \frac{\mu J_{2} a_{e}^{2}}{r^{4}} \sin ^{2} i \sin 2 u \\
F_{w}=-\frac{3}{2} \frac{\mu J_{2} a_{e}^{2}}{r^{4}} \sin i \cos i \sin u .
\end{gathered}
$$

$J_{2}$ is responsible for the secular rates of the right ascension of ascending node and the argument of perigee.

\subsection{Perturbation due to atmospheric drag}

The motion of the satellite in high-altitude atmosphere produces drag force that changes orbital parameters. The rate of orbital decay depends on atmospheric density; this varies with time and geographic position and is not precisely known.

The drag effect on a satellite orbit is expressed through energy dissipation: when an orbit loses energy, its semimajor axis decreases. The drag effect would circularize the orbit - for orbit with large eccentricity, by gradually lowering its apogee until the satellite crashes on the Earth's surface.

For circular orbits, the orbit decay rate can be computed by the following equation:

$$
\frac{d a}{d t}=-\sqrt{\mu a} \rho B C
$$

where $\mu$ is gravitational constant, $a$ is semi-major axis, and $B C$ - ballistic coefficient.

When orbits are low-altitude ones, less than $1000 \mathrm{~km}$, the drag effect should be considered in long-term predictions. The perturbing acceleration due to drag effect could be given as [1]:

$$
a_{D}=-\frac{1}{2} \rho V^{2} \frac{C_{D} A}{m} i_{v}
$$

where $a_{D}$ is atmosphere drag acceleration vector; $\rho$ - air density and parameters of the satellite: $A$ - effective area; $m$ - mass; $V$ - velocity and $i_{v}-$ unit vector of the velocity.

At this point the hardest obstacle is modeling the density of the atmosphere, which is function of time and altitude.

\subsection{Solar radiation pressure perturbation}

The solar-radiation pressure is induced by photons emitted from the sun. For the purposes of the study solarradiation pressure at each point of the orbits is accepted to be a constant. The magnitude of solar-radiation-pressure in one astronomical unit (AU) is $4,65 \times 10^{5}$ dyne $/ \mathrm{cm}^{2}$.

Perturbing acceleration $a_{p}$, due to solar-radiation pressure effect, could be expressed by [1]:

$$
a_{p}=4,65 \cdot 10^{-6}(1+\beta)\left(\frac{A}{m}\right)\left(\frac{a_{\odot}}{r_{\odot}}\right)^{2},
$$

where: $\beta$ is optical reflection constant $(\beta=1$ for total reflection, $\beta=0$ total reception, $\beta=-1$ for total transmission); $A$ - satellite effective area, $\mathrm{m}^{2}, m$ - satellite mass, $\mathrm{kg} ; a \odot$ and $r \odot-$ semi-major axis and radius of the Sun's orbit around Earth; correlation $(a \odot / r \odot) \approx 1$ for near Earth orbits.

\section{Perturbations other than those related with satellite orbits}

\subsection{Satellite clock error}

GPS satellites contain an atomic clock that synchronizes all onboard operations, including the generation and transmission of a signal. The acceptable deviation between satellite time and system time is $1 \mathrm{~ms}$. The MCS determines and transmits clock correction parameters to the satellites for rebroadcast in the navigation message. The bias can be computed in the user/receiver by means of the following [3]:

$$
\delta t_{c l c}=a_{f 0}+a_{f 1}\left(t-t_{o c}\right)+a_{f 2}\left(t-t_{o c}\right)^{2}+\Delta t_{r},
$$

where $a_{f 0}$ is clock bias, s; $a_{f 1}-$ clock drift; $a_{f 2}$ - frequency drift (i.e. aging), s/ $\mathrm{s}^{2} ; t_{o c}$ - clock data reference time, $\mathrm{s}$; $t$-current time epoch, $\mathrm{s} ; \Delta t_{r}-$ correction due to relativistic effects, s.

This residual clock error $\delta t$ results in ranging errors that typically vary from $0,3-4 \mathrm{~m}$.

\subsection{Relativistic Effects}

The need for relativistic correction related to special theories of relativity occurs whenever the GPS satellites and the GPS receivers:

- move with respect to the chosen isotropic light speed frame, which in the GPS system is the ECI frame;

- are located at different gravitational potentials. 
In order to compensate for both of these effects, the satellite clock frequency is adjusted to 10,22999999543 $\mathrm{MHz}$ prior to launch. The frequency observed by the user at sea level will be 10,23 MHz; hence, the user does not have to correct for this effect.

Due to slight eccentricity of the satellite orbit the influence of other relativistic effects must be adjusted, such as periodic change in its gravitational potential. When the satellite is at perigee the velocity of the satellite is higher and gravitational potential lower and both cause delay of satellite clock. On the contrary - when the satellite is at apogee the velocity is lower and gravitational potential is higher. Both make the satellite clock to run faster. This effect could be expressed as [3]:

$$
\Delta t_{r}=F \cdot e \cdot \sqrt{a} \sin E_{k},
$$

where $F=-4,442807633.10^{-10} \mathrm{~s} / \mathrm{m}^{1 / 2} ; e$ - eccentricity; $a$-semi-major axis; $E_{k}-$ eccentric anomaly of the satellite orbit.

Correcting the satellite clock will result in a more precise assessment of the time of propagation of the navigational signal.

\section{3 lonosphere effect}

For the purpose of GPS applications the Earth atmosphere is divided into two sections: the ionosphere and the troposphere. The ionosphere is located between about 70 $\mathrm{km}$ and 1,000 km above the Earth's surface. An ionized environment causes a slow down of the group velocity of the electromagnetic wave and a rise in the carrier phase. This is known as ionospheric effect that is frequency dependent, i.e. it forms $\rho_{L 1}$ and $\rho_{L 2}$ delay. This effect can be eliminated by making ranging measurements with a dual-frequency receiver - pseudorange measurements made on both $f_{L 1}$ and $f_{L 2}$.

To estimate the impact of ionospheric delay on measured pseudorange using $f_{L 1}$ could be expressed as follows [3]:

$$
\Delta S_{\text {iono, } \operatorname{corr}_{L 1}}=\left(\frac{f_{L 2}^{2}}{f_{L 2}^{2}-f_{L 1}^{2}}\right)\left(\rho_{L 1}-\rho_{L 2}\right) .
$$

The path length difference on L2 can be estimated by multiplying $\Delta S_{\text {iono, } \text { corr }_{L 1}}$ by [3]:

$$
\left(f_{L 1} / f_{L 2}\right)^{2}=(77 / 60)^{2} .
$$

These calculated adjustments could be smoothed out over time, since the errors caused by ionospheric delay do not change rapidly and are subtracted from the pseudorange measurements made by each frequency.

When a satellite is at low elevation the signal delay is three times bigger than that at the zenith. For a signal arriving at vertical incidence, the delay ranges from about $10 \mathrm{~ns}(3 \mathrm{~m})$ at night to as many as $50 \mathrm{~ns}(15 \mathrm{~m})$ during the day. At low satellite viewing angles $\left(0^{\circ}\right.$ through $\left.10^{\circ}\right)$, the delay can range from $30 \mathrm{~ns}(9 \mathrm{~m})$ at night to up to $150 \mathrm{~ns}$ (45 m) during the day.

\subsection{Tropospheric delay}

The troposphere is the lower part of the atmosphere that is nondispersive for frequencies up to $15 \mathrm{GHz}$. Phase and group velocity, within this media, related with $\mathrm{C} / \mathrm{A}$ code and GPS navigation data for both signals with frequencies are equally delayed with respect to free-space propagation

The refractivity is often modeled as including both a dry (hydrostatic) and wet (non-hydrostatic) component. The dry component gives about $90 \%$ from tropospheric delay and can be predicted very accurately. It is much more difficult to predict the wet component, which reflects the water vapors, due to the variable nature of the condition of the atmosphere.

One accurate method for modeling the troposphere dry and wet components at zenith without meteorological sensors was developed at the University of New Brunswick (i.e. UNB3 model) [4]. This model is used in the present study.

\subsection{Multipath}

In the present study th emultipath is considered to be constant:

$$
\text { multipath }=\operatorname{tg} 0,25 c / \mathrm{f}_{\mathrm{L} 1} \text {. }
$$

Calculations are made with mask angle range of $5^{\circ}$. $45^{\circ}$, with $5^{\circ}$ step.

\section{Software tool synthesis}

Computation of the errors in position determination, mentioned above, is implemented in computer code. The synthesized software tool structure is shown in Fig. 1.

Three major groups may be distinguished:

- Input module - station2.m;

- Calculation of orbital parameters including orbital perturbations module;

- User position determination module with non-orbital perturbations taken into account as well.

\section{Summary}

The presented algorithm, implemented in a computer code, is a tool that allows for the analysis of the impact a number of factors have when determining the user position; factors such as satellite clock error, relativistic effects, ionosphere effect, troposphere delay, multipath effect, and factors influencing the satellite orbit such as perturbation of gravity, oblateness of the Earth, atmospheric drag, solar radiation pressure, etc.

The synthesized software tool allows to research the impact of perturbation factors on the position errors - one by one, or in various combinations. Numerical simulations were performed as well and the results of the analysis will be presented in a forthcoming paper. 


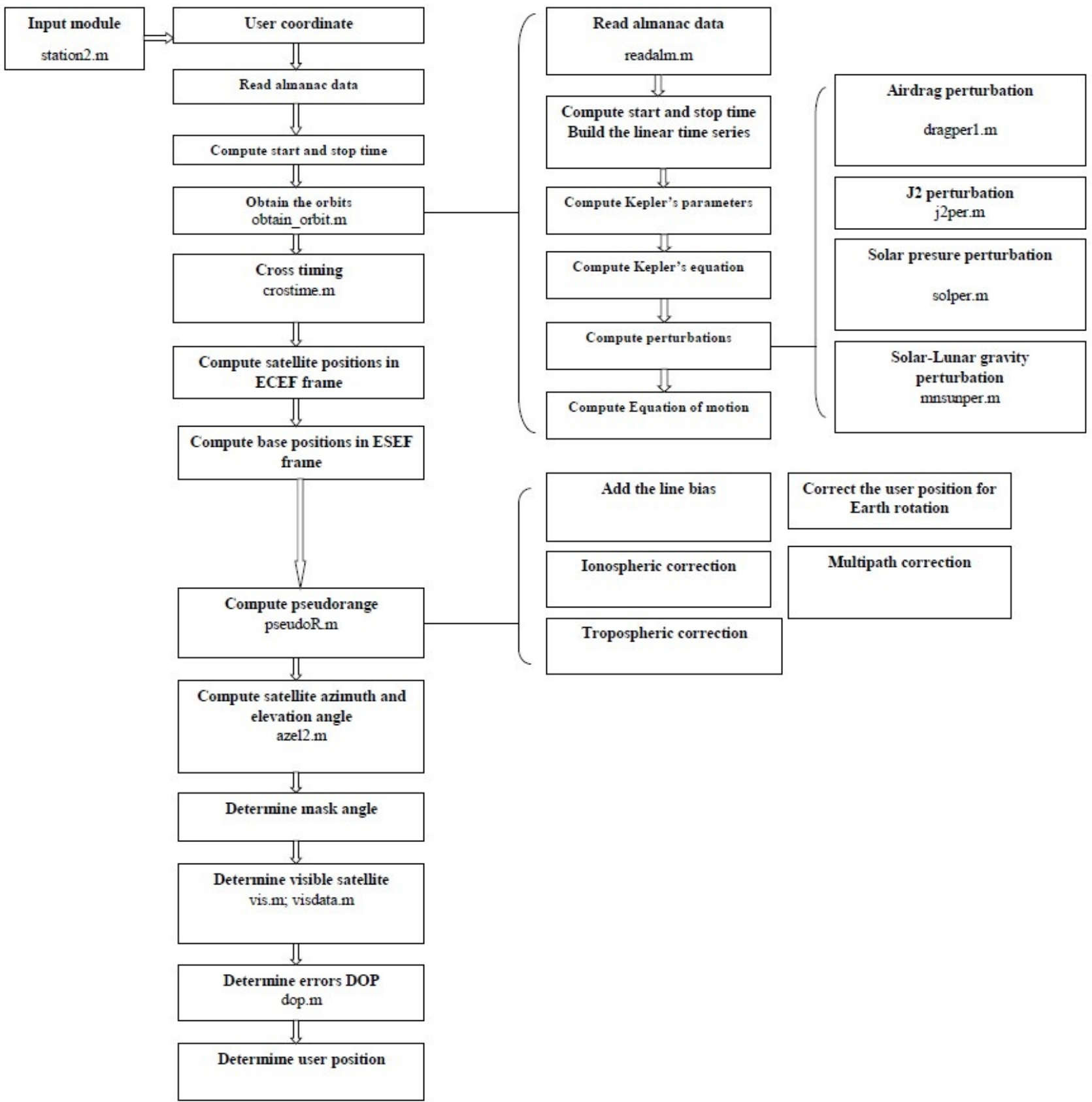

Fig. 1. The software tool structure synthesized.

\section{References}

1. V. Chobotov, Orbital Mechanics, Third edition; American Institute of Aeronautics \& Astronautics, (2002)

2. H. Curtis, Orbital mechanics for engineering students, Elsevier Butterworth-Heinemann, (2005)

3. E. Kaplan, C. Hegarty, Understanding GPS: Principles and applications, Second edition; Artech house, (2006)

4. J. Collins, R. Langley, Nominal and extreme error performance of the UNB3 tropospheric delay model, Technical Report, University of New Brunswick, (1999) 